\title{
Adenoma hipofisiario productor de somatotrofina en una paciente con antecedente de struma ovarii carcinoide
}

\author{
Erick Riedemann S, Claudio Rojas C, Iván Q uevedo L. \\ Somatotropic pituitary tumor in a \\ patient with a previous ovarian \\ strumal carcinoid. Report of one case
}

Strumal carcinoid is an unusual form of monodermal ovarian teratoma with thyroid-like follicles mixed with typical carcinoid tumor patterns. We report a 49 years old woman presenting with an acromegaly. At the age of 45, an ovarian strumal carcinoid was excised and at three years of follow up, she complained of finger and feet growth. Laboratory showed an elevated serum IGF-1 and a sella turcica magnetic resonance imaging showed an $8 \mathrm{~mm}$ microadenoma. She was subjected to a transphenoidal excision and the pathological study disclosed a solid acidophilic pituitary adenoma. Two months after surgery, there was a significant decrease in serum IGT-I levels (Rev Méd Chile 2004; 132: 857-9).

(Key Words: Acromegaly; Carcinoid tumor; Pituitary neoplasms)

Recibido el 5 de noviembre, 2003. Aceptado en versión corregida el 6 de mayo, 2004.

Sección de Endocrinología, Departamento de Medicina Interna, Facultad de Medicina, Universidad de Concepción.

$\mathrm{E}^{1}$ struma ovarii es un tumor quístico de los ovarios, que corresponde aproximadamente al $0,4 \%$ de los tumores ováricos ${ }^{1}$. El tejido tiroideo del struma ovarii es funcional y estructuralmente similar al tejido tiroideo cervical normal. Generalmente se presenta como un tumor ovárico asintomático ${ }^{2}$. Una forma inhabitual de struma ovarii es el carcinoide del estroma, en que existe la presencia de tejido tiroideo con tumor carcinoide en un tumor ovárico monodermal ${ }^{3}$. Clínicamente la asociación con ascitis es relativamente común y puede ocurrir en más del $10 \%$ de los $\operatorname{casos}^{4,5}$.

Correspondencia a: Dr. Iván Quevedo L Avenida Andalué No 1825, San Pedro de la Paz, Concepción - Chile.

E-mail: equevedo@udec.cl
Al revisar la literatura, sólo existe un caso documentado de asociación de struma ovarii y adenoma hipofisiario, que comespondía a un microprolactinoma ${ }^{1}$. Hasta donde sabemos, no se ha reportado ningún caso de struma ovarii en pacientes con adenoma hipofisiario productor de somatotrofina.

Presentamos el caso de una mujer que en el seguimiento de una struma ovarii (carcinoide del estroma) se detectó un adenoma hipofisiario secretor de somatotrofina.

\section{CASO CLÍNICO}

Mujer de 49 años, previamente asintomática, sin antecedentes mórbidos de importancia, con dos 
hijos sanos de partos normales, que refenía reglas regulares hasta los 45 años. A esa edad presentó dolor cólico en flanco derecho que se irradiaba al resto del abdomen. Dado que el dolor se hizo persistente, consultó a ginecólogo que encontró en el examen fondos de saco vaginales ocupado por un tumor intrapélvico. Tenía hemograma, examen de orina, creatininemia y glicemia normales. Se le realizó ecotomografía de abdomen y pelvis que mostró un tumor quístico gigante abdómino pelviano, de probable origen ovárico. La ecotomografía ginecológica transvaginal mostró una masa en la zona anexial izquierda de 107 x 74 x $108 \mathrm{~mm}$ y líquido libre en el fondo de saco de Douglas. Fue intervenida quirúrgicamente por laparotomía media infraumbilical, encontrándose en la cirugía 6 litros de líquido hemorrágico en el peritoneo y un gran quiste ovárico izquierdo. El páncreas tenía una estructura normal. La biopsia concluyó que el tumor correspondía a un teratoma ovánico monodermal con tejido tiroideo y tumor carcinoide clásico (estroma carcinoide) (Figura 1). El líquido peritoneal no mostró células neoplásicas. Fue controlada, posteriormente, cada 6 meses con ecotomografía ginecológica. La paciente refirió a los tres años, crecimiento del diámetro de los dedos de ambas manos y la talla del zapato. Dado lo anterior, fue derivada a endocrinólogo, por sospecha de acromegalia. En la evaluación endocrinológica destacaba historia de tres años de crecimientos de las partes blandas de los pies y de las manos. Al examen físico era evidente fascie acromegálica y macroglosia: timoides era de forma y tamaño normales. El estudio hormonal mostró IGF-I elevado $690 \mathrm{mg} / \mathrm{mL}$ (VR: 70-554) e hiperproducción autónoma de hormona de crecimiento (Tabla 1). La tomografía axial computarizada y resonancia nuclear magnética de silla turca mostraron un microadenoma de $8 \mathrm{~mm}$ de diámetro, sin compromiso del

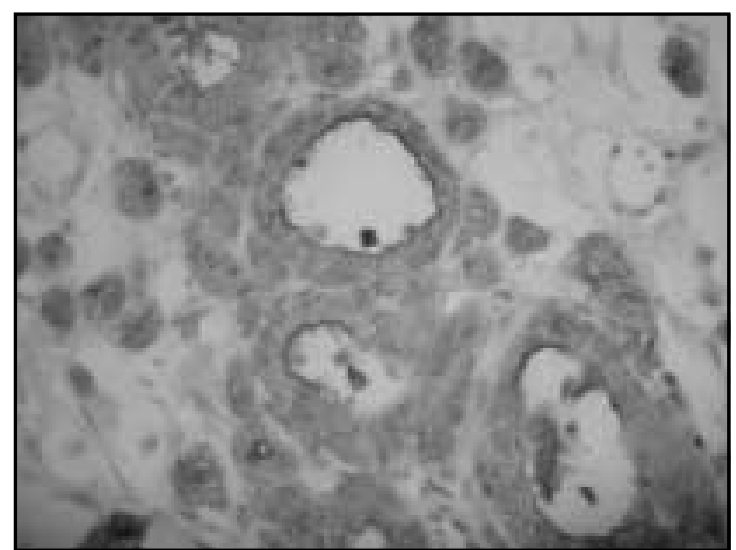

FIgURA 1. Biopsia de tumor quístico ovárico donde se aprecia tejido tiroideo en un teratoma ovárico.

quiasma óptico ni senos cavernosos (Figura 2). Se realizó exéresis transesfenoidal sublabial del microadenoma. No tuvo complicaciones postoperatorias. La biopsia mostró adenoma hipofisiario de tipo sólido y acidófilo. El control hormonal, realizado a los dos meses, mostró una significativa disminución de IGF-I y de los niveles plasmáticos de GH en el test de sobrecarga de glucosa (Tabla 1).

\section{DisCUSIÓN}

El hallazgo de tejido tiroideo fue descrito por primera vez en $1889^{6}$. Se describe la presencia de tejido tiroideo en $0,6 \%$ de los teratomas ${ }^{1}$. Sin embargo, el diagnóstico de struma ovarii requiere que el tejido tiroideo sea el constituyente principal del tumor, o que sintetice suficiente hormona tiroidea como para producir hipertiroidismo ${ }^{1}$. En el caso de nuestra paciente, aproximadamente la mitad del tumor quístico correspondía a tejido

Tabla 1. Evaluación bioquímica pre y post quirúrgica de acromegalia con niveles plasmáticos de IG F-I y test de supresibilidad de hormona de crecimiento con carga oral de $75 \mathrm{~g}$ de glucosa

\begin{tabular}{|lcccc|}
\hline & \multicolumn{2}{c}{ Preoperatorio } & \multicolumn{2}{c|}{ Postoperatorio } \\
& Glicemia $(\mathrm{mg} / \mathrm{dl})$ & GH $(\mathrm{ng} / \mathrm{ml})$ & Glicemia $(\mathrm{mg} / \mathrm{dl})$ & $\mathrm{GH}(\mathrm{ng} / \mathrm{ml})$ \\
\hline Basal & 95 & 4,3 & 88 & 2,8 \\
30 minutos & 144 & 3,4 & 134 & 1,8 \\
60 minutos & 208 & 3,6 & 125 & 1,5 \\
120 minutos & 137 & 4,3 & 108 & 1,2 \\
IGF-I & 690 & - & 291 & - \\
\hline
\end{tabular}




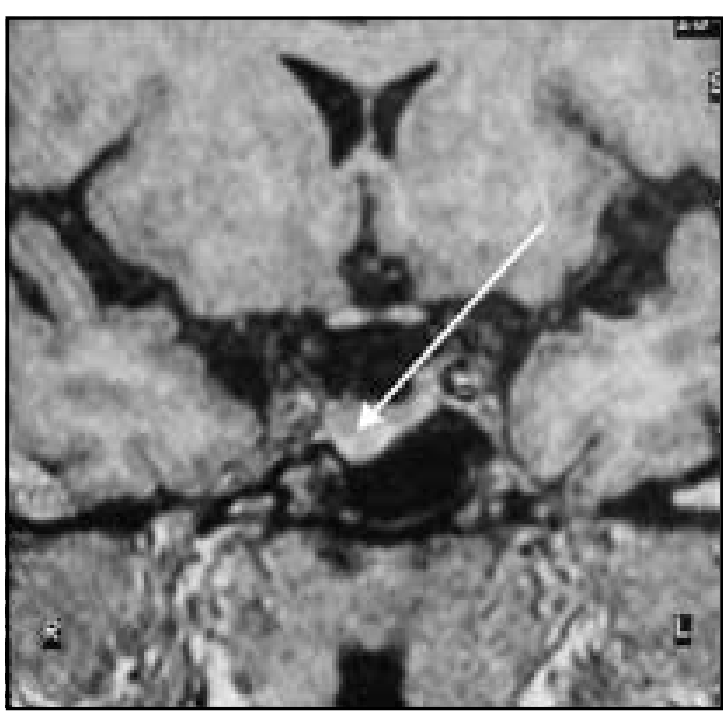

FIgURA 2. Resonancia nuclear magnética de silla turca que evidencia microadenoma de $8 \mathrm{~mm}$.

\section{REFERENCIAS}

1. Dunzendorfer T, De Las Morenas A, Kalir T, Levin R. Struma ovarii and Hyperthyroidism. Thyroid 1999; 9: 499-502.

2. KeMPERS RD, DOCKERTY MB, HOFFMAN DL, BathoLOMEW LG. Struma ovarii-Ascitic, hyperthyroid, and asymptomatic syndromes. Ann Inter Med 1970; 72: 883-98.

3. Tamsen A, Mazur MT. Ovarian Strumal Carcinoid in association with multiple endocrine neoplasia, type II A. Arch Pathol Lab Med 1992; 116: 200-3.

4. Szyfeibein YM, Young RH, ScuLy RE. Struma ovarii simulating ovarian tumors of the other types. A report of 30 cases. Am J Surg Pathol 1995; 19: 21-9.

5. Bethune M, QuinN M, Rome R. Struma ovarii presenting as acute pseudo-Meigs syndrome with an elevated CA 125 level. Aust N Z J Obstet Gynecol 1996; 36: 372-3.

6. BoEtTun R. Ueber Zahnentwicklung in Dermoidzystem des Ovariums. Virchows Arch Path Anat 1889; 115: 493-504.

7. Lin FC, Lin CM, HsieH CC, L WY, WANg LS. Atypical Thymic Carcinoid and Malignant Somastotinoma in type I multiple endocrine neoplasia syndrome. Case report. Am J Clin Oncol 2003; 26: 270-2.

8. Boix E, Pico A, Pinedo R, Aranda I, Kavacs K. Ectopic Growth Hormone-Releasing Hormone Secretion by Thymic Cancinoid Tumor. Clin Endocrinol (Oxf) 2002; 57: 131-4. tiroideo. Algunos tumores carcinoides, principalmente de timo y próstata, se han reportado en pacientes con neoplasias endocrinas múltiples ${ }^{7-11}$. Nuestra paciente en el estudio imagenológico y bioquímico no evidenció neoplasia de tiroides, paratiroides, páncreas o suprarrenales.

La presencia de struma ovarii y adenoma hipofisiario sólo ha sido reportada en una ocasión en relación a una paciente con microprolactinoma ${ }^{1}$. Sin embargo, el hecho que nuestra paciente haya tenido tejido carcinoide concomitante con tejido tiroideo, podría relacionarla con una etiopatogenia común con los casos de neoplasias endocrinas múltiples tipo I en pacientes que además tenían carcinoides de timo ${ }^{7,9,10-12}$ o duodeno ${ }^{13,14}$. Hasta el momento, en el seguimiento clínico y bioquímico de nuestra paciente, ni en el seguimiento de sus dos hijos, se han evidenciado hallazgos que orienten a neoplasia endocrina múltiple.

9. Hirai S, Hamanaka Y, Mitsui N, Kumagai H, KobayasHI T. Thymic carcinoid in multiple endocrine neoplasia-Type I. Jpn J Thorac Cardiovasc Surg 2001; 49: 525-7.

10. Sugiura $H$, Morikawa T, Iтoh $K$, Ono K, O kushiba $S$, Kondo S, Kato H. Thymic carcinoid in patient with multiple endocrine neoplasia type I: Report of a case. Surg Today 2001; 31: 428-32.

11. Whelan T, Gatfield CT, Robertson S, Carpenter B, SCHILNGER JF. Primary carcinoid of the prostate in conjunction with multiple endocrine neoplasia II B in a child. J Urol 1995; 153: 1080-2.

12. The BT. Thymic carcinoids in multiple endocrine neoplasia type I. J Intern Med 1998; 243: 501-4.

13. Yazawa $K$, Kuroda $T$, Watanabe $H$, Shimozawa $N$, NimURA Y, NAKATA S ET aL. Multiple carcinoids of the duodenum accompanied by type I familial multiple endocrine neoplasia. Surg Today 1998; 28: 636-9.

14. Tokumitsu $M$, Yamaguchi $S$, Noda T, Numata $M$, MoriKaWA M, Mirata M Et al. A case of multiple endocrine neoplasia type I with primary hyperparathyroidism, prolactin secreting pituitary microadenoma and gastrin secreting duodenal carcinoid. J Nucl Med 1988; 29: 263-5.

Agradecimiento

Los autores agradecen la valiosa colaboración en la redacción y ordenamiento de la Sra. Ana Cristina Contreras, Secretaria Centro Saval de Concepción. 